\title{
Distributed Resource Reservation for Beacon Based MAC Protocols
}

\author{
Frank Leipold ${ }^{1}$ and Jörg Eberspächer ${ }^{2}$ \\ 1 Sensors, Electronics and Systems Integration \\ EADS Innovation Works Germany \\ frank. leipold@eads.net \\ 2 Institute of Communication Networks \\ Technische Universität München \\ joerg.eberspaecher@tum.de
}

\begin{abstract}
Wireless connected devices become increasingly popular in a large variety of applications. Consumer electronics most certainly is the field with the most wireless innovations in the past years; but also other areas, such as medical equipment, vehicular on-board networks or maintenance services, experience an increasing demand for wireless communication. Additionally the networks should just work and require as little maintenance as possible. Hence future WLAN and WPAN must be self-configuring, self-healing and distributed to provide flexible usage.

Assuming a homogeneous distributed MAC protocol with a beacon based reservation mechanism, a radio resource reservation algorithm is developed to fulfil the delay and data rate requirements from the devices. It uses a game theoretic approach to achieve infrastructure-less design and still provides fair resource allocation. Changes in the radio channel, failing devices or links and mobile nodes are detected and a reorganisation of resources is calculated.
\end{abstract}

\section{Introduction}

The constantly increasing performance demands for wireless high speed connections is the motor to further enhance existing technologies and to invent new and innovative communication interfaces. For wireless local area networks (WLAN) several candidates have been developed in the past years. The recently approved IEEE 802.11n standard achieves up to $600 \mathrm{Mbits} / \mathrm{s}$, by using MIMO (multipleinput-multiple-output) technology.

High data rates can also be easily achieved by using ultra wideband (UWB) as the bandwidth of more than $500 \mathrm{MHz}$ per channel provides plenty resources. The WiMedia standard 1 is capable of $480 \mathrm{Mbit} / \mathrm{s}$ with one single channel. The recently published upgrade even allows $1024 \mathrm{Mbit} / \mathrm{s}$. This is done with one single antenna (hence no MIMO), which implies less complex transceivers.

One significant difference between WiFi and high-speed UWB is the transmit range. Due to the strict power limitations of at most $-41.3 \mathrm{dBm} / \mathrm{MHz}$ for $\mathrm{UWB}$, defined from the FCC [2, and mostly followed by other regulatory organisations, 
high data rate UWB has a typical range of about 10 meters. It also underlies more intense spatial limitations given by walls and other obstacles. This is often interpreted as a handicap of UWB, but it actually also implies two significant advantages. First it reduces the congestion in the wireless channel. For instance the IEEE $802.11 \mathrm{~b} / \mathrm{g}$ technology is currently often used in home applications. But the standard has only three non-overlapping channels (respectively four in Japan). Thus in apartment houses, where most flats have their own WiFi access point (AP), the channels are very crowded as the technology can achieve 20 to 100 meters transmit range even in indoor scenarios. With UWB the radio signals are almost confined to the individual apartments and the contentions for the wireless channels get relaxed. There will be less wireless systems sharing the common resources. The second advantage is the reduced risks of being eavesdropped. The attacker must be in very close proximity to successfully receive the UWB signals. Eavesdropping becomes much more difficult and the risk of falling victim to an attack declines.

Even though WiMedia is a fully distributed algorithm, without any coordinating nodes such as a WiFi-access-points, the network still has service providing access points, for instance a node that is connected to a LAN and operates as a bridge between the LAN and the WiMedia network to enable access to the Internet. These WiMedia (or UWB) APs do not have any special role in terms of the PHY or MAC protocol.

Using UWB for WLAN applications requires a larger number of APs. For the office scenario about each room requires at least one AP, depending on the size of the room. This makes the network management more complex. For now usually the WiFi networks are managed by maintenance personnel, but with a large number of APs to manage, this becomes a very complicated task. Several automated resource management algorithms for WLAN systems have been proposed yet. For WiMedia little work has been done on this subject. With its completely distributed algorithms the existing approaches can not be used efficiently.

In an earlier work 3 an integer optimization algorithm was presented to calculate the optimal AP placement and resource allocation plan for WiMedia networks. It is very complex and time consuming algorithm that will run during the design phase of the network. But during the operation of the network changes to the wireless channel will occur and a fast adaptation to the new conditions must be made. Depending on the size of the network a centralised optimisation may take too long. A distributed approach is preferred.

Our applications target wireless on-board networks for public transportation vehicles, such as aircraft. Cabin management systems, containing reading lights, signs, speakers, small displays, shall be connected wireless to reduce production and maintenance costs and to make the cabin layout more flexible. UWB currently is the most promising technology that provides a very robust radio channel for the aircraft environment [4, high data-rates and no licensing problems. Furthermore the network shall have self-healing capabilities and adapt 
quickly to failures and changes. Therefore the resource management algorithm should be distributed and enable the network to operate with the best possible configuration even when some parts are failing completely.

The network management of the public transportation scenario is not very different from an office environment or home consumer electronics. Therefore the gained results in this paper can be easily applied to other situations, where devices must be connected to service access points.

The rest of this paper is organised as followed. Section 2 describes features of WiMedia relevant to this work. Section 3 describes briefly game theory and some related research. In section 4 the distributed resource management algorithm is presented. Finally section 5 concludes this paper.

\section{Unique WiMedia Features}

The WiMedia standard and also other possible standards that use a distributed beacon based MAC implementation, has some unique features, which are of value for the resource management.

WiMedia is completely infrastructure-less, meaning all nodes have the same physical and MAC capabilities. Unlike in other common protocols there is no coordinator or MAC level access point. The protocol uses periodic superframes containing a beacon period and a data period; see Figure 1(a). All nodes allocate a beacon slot in the beacon period, regardless if they have to transmit user data or not. This slot is fix over time and changes only in rare conditions. For DRP (distributed reservation protocol) channel access the beacons are used to announce transmissions and reserve parts of the data period, the so called MAS (media access slots). Conflicts are identified early and collisions can be mostly avoided. The only collisions may occur in the beacon slot allocation process.

The beacons contain details of all upcoming transmission in the data period. This means a single node knows when a neighbour communicates with another node. This feature makes WiMedia not susceptible to the hidden station problem and it does not need RTS/CTS messages.

With the beaconing a hard limitation of WiMedia applies. The beacon period has only 96 beacon slots with two slots reserved for special functions. This means no more than 94 nodes must be within range on the same channel. The group of nodes within transmit range of a subject node is called beacon group (BG). Each node includes the members of the BG and the respective beacon slot ids into its own beacon; hence it is broadcasting this information to the surrounding nodes. Furthermore the standard also defines the extended beacon group (EBG), which is the set of nodes representing the neighbour's neighbours. Figure 1(b) shows the BG and the EBG of a node. A newly activated node picks a beacon slot that is not occupied by a node of the BG nor from the EBG. This rule implies that for any given node the beacon slot ids of the BG members must be unique, otherwise they would cause collisions. For the EBG members the ids can be identical, because the subject node can not listen to them, but must not 


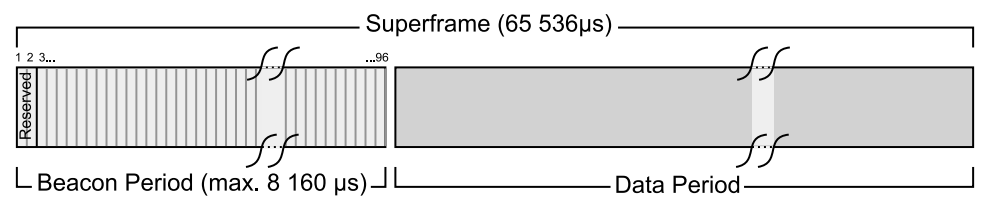

(a) Superframe structure

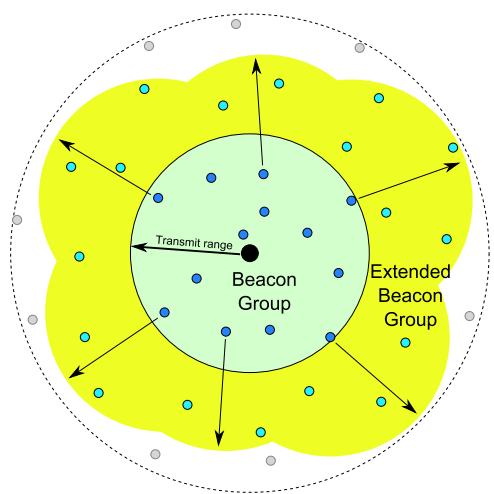

(b) Beacon group and extended beacon group

Fig. 1. WiMedia beacon groups: Figure (a) shows the beacon period and data period of a superframe. In (b) the relation of the beacon group and the extended beacon group are shown.

transmit at the same time as it would cause collision at the node that has the subject node and the EBG-node in its BG.

The consequence of overlapping areas in WiMedia is the extension of the EBG. When a border node is within range of two APs, it will add parts of the second AP nodes to its BG, which again appear in the EBG of the first AP. Hence overlaps increase the extended beacon group size and therewith limit the maximal node density.

The beacon group size is directly related to the node density and transmission range as slots may only be occupied by one node. The extended beacon group size additionally depends on the activation sequence of the network, as for a single node the neighbour's neighbours beacon slots may be identical.

\section{Game Theory for Resource Management}

For the distributed resource management algorithm presented in this work, one key feature is to have a fair resource allocation. Nodes should not act selfishness and occupy wastefully available resources. The objective of the algorithm is to maximize the overall efficiency of the network. Hence nodes must not only take their own situation into account, but also those of the surrounding nodes. 
The game theory is capable of defining fair and distributed algorithms. It originates from economics in the 40's, but has also been applied to biology, engineering, political science, computer science, and philosophy. The problem is defined in a strategic game with a set of rational players. Each player tries to maximise its payoff function by choosing the best strategy and also by taking the actions of the other players into account. Usually the players strategies will result in an equilibrium, for instance the Nash equilibrium, where no player can change the own strategy without decreasing its payoff. Many different types of games have been developed: cooperative or non-cooperative, symmetric or asymmetric, zero-sum or non-zero-sum, simultaneous or sequential, just to name a few.

Game theory has been proposed for resource management in wireless networks for all kinds of technologies. For IEEE 802.11 the authors of [5] present a game to share the available radio channels. The payoff function based on transmission delay, channel access length and throughput. In [6] an algorithm for OFDM based communication is described that minimizes the transmission power, while still achieving the QoS requirements of the system. For IEEE 802.16 networks 7. presents a game definition to control the amount of bandwidth given to new connections, with respect to delay, throughput and other QoS parameters in the network.

Even though the amount of available resource management algorithms based on game theory is huge, no one handles a comparable system to that of a WiMedia network. The distributed beaconing mechanism and DRP channel access scheme is unique. The priority for a WiMedia network lies in the efficient MAS allocation. To utilise the WiMedia features a new resource management algorithm is required.

\section{Algorithm}

The resource management algorithm assumes APs with fix locations. Furthermore the network consists of a wired backbone that connects the APs. The APs provide the service for wireless end devices to get access to the wired back bone network.

The MAC mechanism for the WiMedia nodes shall be DRP. For the aircraft scenario DRP is essential, as it provides guaranteed resources to the end devices. The QoS requirements for end devices are defined in required data rate and maximum time delay. Assuming only one MAS is reserved per end device and taken into account the $64 \mathrm{~ms}$ of a superframe, the delay of an message over the UWB link can be nearly $64 \mathrm{~ms}$. For applications with smaller delay requirements, the resource management algorithm must reserve two or more MAS per end device and per superframe with defined maximum gaps between the MAS.

Despite the mentioned DRP reservation scheme the network may also use the alternative channel access PCA (prioritized contention access) for non critical applications. MAS that are not completely used by the owing device can be released and used for PCA, a CSMA like access scheme. This way the unused MAS sections can still be used for applications where collisions can be tolerated. 
The following two lists show input and output parameters of the algorithm:

\section{Input parameters}

- Link quality

- Devices per AP

- BG/EBG size

- Bandwidth utilisation

- Delay requirements
Output parameters

- Channel allocation

- MAS reservation

- Beacon slot reservation

The link quality is given in RSSI (received signal strength indicator) or LQE (link quality estimator). Both are defined in the WiMedia standard and should be accessible from the application layer. To provide the required information of the surrounding nodes, the QoS requirements, neighbourhood relation and currently superframe structure is exchanged on a two-hop distance. This is mostly already present in the WiMedia protocol and requires only little extra signalling.

Efficiency in a WiMedia network using DRP can be defined as the configuration with the minimal used MAS, which again implies the minimal usage of resources and maximum remaining bandwidth. The straight forward approach then would be to choose the AP only by signal quality. A node would therefore always try to connect to the AP with the best signal. But for an unlucky network topology, as is Figure 2, this could mean that many nodes try to connect to the same AP while a second AP close by is ignored, because it is further away. If these are too many nodes, the network performance decreases at this AP. The goal of the algorithm is to enable some nodes to choose a different AP, if the actually best AP has a lot of load.

From the protocol perspective two limiting factors exist. The first one is the number of beacon slots. As described earlier, only 94 slots are available for the BG and the EBG. Not more than 94 nodes can be simultaneously in transmit range. To allow new nodes to connect to the network, a small amount of slots should be kept unoccupied.

The second limiting factor is the number of MAS. 256 MAS exist in one superframe, which must be shared among the nodes in transmit range. The

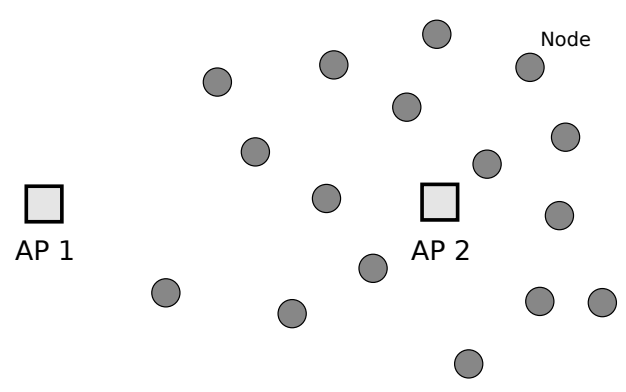

Fig. 2. Network topology where most nodes would try to connect to the same AP, if the selection is only based on signal quality or distance 
MAS reservation depends on the data rate and delay requirements. Again a few MAS should be constantly available to allow new nodes to join the network.

In contrast to the MAS slots stands the data rate between an AP and an end device. An increased data rate requires less MAS reservations and enables more nodes to join the network.

The reservations between an end device and AP are made from the end device. It periodically calculates the utility function $U_{n}$ for all AP in range. It is defined as:

$$
U_{n}=B_{n}^{*}+M_{n}^{*}+D_{n}^{*}
$$

where $B_{n}^{*}$ contains the beacon slot utilisation, $M_{n}^{*}$ the MAS slot utilisation and $D_{n}^{*}$ the possible data rate. All three values are normalised to values between 0 and 1 , where 0 means the best achievable value and 1 the worst value. Each end device periodically calculates the utility value for each AP and initiates an AP change, if a better solution was found than the currently one.

$B_{n}^{*}$ is defined as the number of reserved beacon slots $b_{a}$ at the AP $a$, divided by the maximal number of beacon slots $b_{\max }$ to the power of $v$ :

$$
B_{n}^{*}=\left(\frac{b_{a}}{b_{\max }}\right)^{v}
$$

Analogous to this, $M_{n}^{*}$ is defined as:

$$
M_{n}^{*}=\left(\frac{m_{a}}{m_{\max }}\right)^{v}
$$

where $m_{a}$ are the number of reserved MAS at the AP and $m_{\max }$ the number of maximal available MAS slots. $m_{a}$ is obtained by calculating the own number of required MAS, depending on the delay and data rate requirements, and adding it with the already used MAS by other nodes. The MAS reservation algorithm.

$B_{n}^{*}$ or $M_{n}^{*}$ describe the utilisation of the beacon slots or MAS. They should only get a value close to 1 if the number of utilised slots becomes close to the maximum. By modifying $v$ one can control the threshold.

Finally $D_{n}$ is a linear relation of the possible data rate between the end device and the $\mathrm{AP} d_{n, a}$ and the maximal possible data rate $d_{\max }$ :

$$
D_{n}^{*}=\left(1-\frac{d_{n, a}}{d_{\max }}\right) * T
$$

$D_{n}^{*}$ must degrade over time if $B_{n}^{*}$ or $M_{n}^{*}$ are above the threshold. Therefore $T$ is used; it usually has a value of 1 , unless the threshold is reached. Then $T$ will increase for each round (when the utilisation values are recalculated), until the node has changed the AP or $B_{n}^{*}$ and $M_{n}^{*}$ drop below the threshold, due to other nodes disconnecting from the AP. With this feature one can achieve to let the nodes closest to the other AP (in Figure 2) first change the AP. $D_{n}^{*}$ for the overloaded AP increases for all nodes in its range. The nodes close to the other AP will have a relative low $D_{n}^{*}$ for the less used AP, because they are closer. They will be the first to change. 
All three addend in Equation 1 are influenced by the current reservations of surrounding nodes. The algorithm considers the actions of the other nodes and derives its own actions. After some rounds an equilibrium will be found where each node can not improve its situation.

For the APs themselves only the channel selection and beacon slot reservation must be done. For them the procedure is to choose a channel that is not used by another AP in its BG or EBG. If no free channel can be found the AP should choose the channel with the least nodes assigned to it.

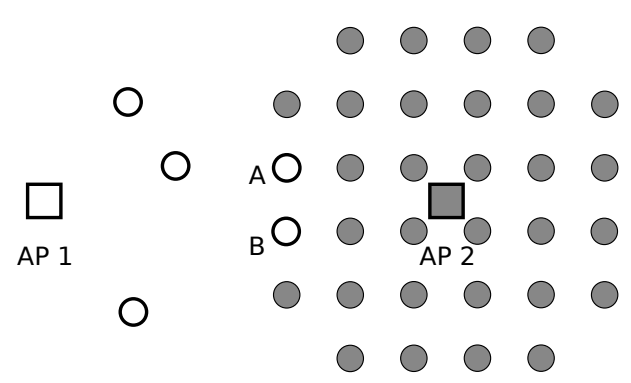

Fig. 3. Final results of the algorithm, after 9 rounds. The transmit range is larger than the show field; hence all nodes are in range of each other. The filled nodes are on one channel and the unfilled nodes on another one. Nodes A and B, close to the left AP, initiated an AP change after 8 round.

In Figure 3 the results of the algorithm are shown. To demonstrate the results in a smaller scale, the maximal beacon slots number and MAS were set to smaller values as defined in the standard. After the first round of calculating the utilisation values, each node chose the closest AP. This resulted in a situation where the threshold for the beacon slots was exceeded. Hence for the following rounds the parameter $T$ for calculating the utilisation of AP 2 increased, until the utilisation value of AP 1 for nodes A and B became smaller as for AP 2 . After node A and B having changed the AP, the threshold is no longer exceeded and the network is in an equilibrium.

\section{Conclusions}

In this work a resource management algorithm based on game theory for high data rate UWB networks was presented. The network consists of service access points, which provide access for wireless end devices to a wired network, such as LAN or Internet. The algorithm achieves a fair distribution of resources and adapts to changes in the radio channel or topology. Network efficiency is based on the number of used MAS slots, which also encompass bandwidth and timing requirements. The MAS reservation algorithm is essential for scenarios with high MAS utilisation, as the efficiency of the overall algorithm is tightly coupled to the efficiency of the MAS reservation for large loads. 
The distributed approach does not require a dedicated node to compute the network parameters. Hence whatever parts of the network are failing, the network still tries to connect as many nodes to the APs as possible. The algorithm can be used in various applications: on-board networks, office and home WLAN or ad-hoc communication.

\section{References}

1. ECMA International, ECMA-368: High Rate Ultra Wideband PHY and MAC Standard, 3rd edn. (December 2008)

2. FCC, Report and Order. FCC 02-48 (April 2002)

3. Leipold, F., Bovelli, S.: Requirements for Radio Resource Management in Multi-cell WiMedia Networks. In: ICT-MobileSummit (2009)

4. Chuang, J., Xin, N., Huang, H., Chiu, S., Michelson, D.: UWB Radiowave Propagation within the Passenger Cabin of a Boeing 737-200 Aircraft. In: IEEE 65th Vehicular Technology Conference, VTC 2007-Spring (April 2007)

5. Berlemann, L., Hiertz, G., Walke, B., Mangold, S.: Radio resource sharing games: enabling QoS support in unlicensed bands. IEEE Network 19 (July-August 2005)

6. Han, Z., Ji, Z., Liu, K.: Power minimization for multi-cell OFDM networks using distributed non-cooperative game approach. In: IEEE Global Telecommunications Conference, GLOBECOM 2004, November-3 December 2004, vol. 6 (2004)

7. Niyato, D., Hossain, E.: Radio resource management games in wireless networks: an approach to bandwidth allocation and admission control for polling service in IEEE 802.16. IEEE Wireless Communications 14 (February 2007) 